\title{
TRUCOS EN PRÓSTATA
}

\author{
José Vicente Rodríguez
}

Fundación Puigvert Barcelona. Barcelona. España.

\section{ÍN DICE/ RESUMEN}

Trucos en:

1. En apertura de senos venosos.

J. Vicente Rodríguez.

2. En erección peroperatoria (anestésica).

J. Vicente Rodríguez.

3. En prevención de la esclerosis cervical post-RTU de próstata.

J. Vicente Rodríguez.

4. En prevención de la eyaculación retrógrada post-RTU de próstata.

J. Vicente Rodríguez.

5. En prevención de la incontinencia yatrógena post-RTU de próstata.

J. Vicente Rodríguez.

Palabras clave: Trucos en próstata. Apertura senos venosos. Erección peroperatoria. Esclerosis cervical Yatrogéna. Eyaculación retrógrada. Incontinencia Yatrógena.

José Vicente Rodríguez

Fundación Puigvert Barcelona.

C/ Cartagena 340-350

08025 Barcelona (España)

\section{TRUCOS EN APERTURA DE SENO VENOSO}

J. Vicente Rodríguez

El sangrado operatorio en la RTU prostática fluctúa entre el $8.6 \%(1)$ y el $2.2 \%(2)$; en nuestra experiencia: 5.3 (3).

Es dependiente de varios factores: dominio técnico, tipo de técnica realizada, gramos resecados, tiempo quirúrgico, pero también del tipo de sangrado. El sangrado arterial es fácilmente identificable y se coagula con facilidad si los cortes de sección son "limpios" y la resección ordenada.

Los senos venosos son difícilmente reconocibles y su apertura acontece al final de la resección

FIG URA 1.
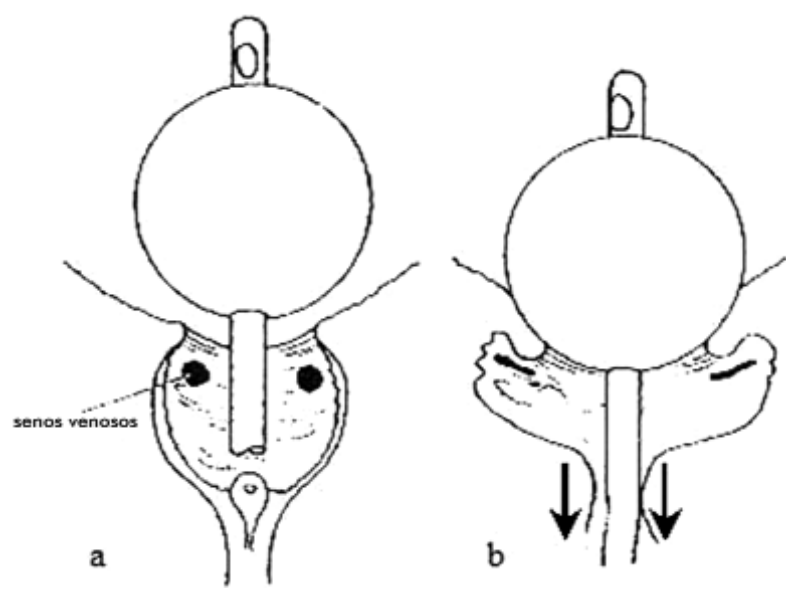
cuando disminuye la presión cavitaria. Los senos venosos se identifican como estructuras tubulares de paredes finas, con sangrado babeante oscuro, que desaparece cuando se lanza sobre él el chorro irrigador. El sangrado venosos es más frecuente en la técnica de inicio anterior, al lesionar los senos antero laterales durante el primer tiempo de la misma.

Para solucionar el sangrado venoso, no se debe coagular directamente el seno, sino realizar un cerclaje de coagulación (con asa de bola) a su alrededor; a veces se consigue la hemostasia, pero solo en senos venosos pequeños.

El "truco" para yugular el sangrado por apertura de seno venoso viene representado en la Figura 1.

Como los senos venosos son abiertos e identificados generalmente en los planos profundos y al final de la intervención (excepto en la técnica de inicio anterior), se debe concluir rápidamente la intervención, colocar sonda uretral con balón de 40-50 mls (a) y traccionar de la sonda (colocar un Salvaris presionando sobre meato uretral con peso conseguido por bolsa de suero de 200-300 mls) durante unos diez minutos (3). La compresión de balón sobre cuello vesical consigue la oclusión del seno venosos y en consecuencia la hemostasia (b).

\section{BIBUOGRAFÍA RECOMENDADA}

1. MADERSBACHER, S.; MARBERGER, M.: "Transurethral resection of prostate still justified?". BJU Int., 83: 227, 1999.

2. ROEHRNORN, C.G.: "Standart surgical interventions: TUIP/TURP/PSU". Textbook of benign prostatic hiperplasya. Eds. ISIS Oxford. 1996.

3. VICENTE, J.: "Tratamiento Endoscópico: RTU / Incisión prostática". HBP 2001 Ed. Vicente. Editorial Acción Médica, 153, 2001.

\section{TRUCOS EN ERECCIÓN PEROPERATORIA (ANESTÉSICA)}

\section{J. Vicente Rodríguez}

La erección o pseudopriapismo preoperatorio es muy poco frecuente, con mayor incidencia postraquianestésica y que constituye un serio inconveniente para la realización de la cirugía transuretral.
La anestesia intradural, que representó el $86.2 \%$ en nuestra experiencia clásica (1), modifica los mecanismos de neurotransmisión y además los cuerpos cavernosos se comportan de forma activa ante estímulos neurológicos (preferentemente adrenérgicos); esos mecanismos en parte desencadenan la erección mantenida entre el 5-10 minutos después de la anestesia raquídea. Este fenómeno es poco frecuente: $0,53 \%$ en nuestra serie clásica (1) y en la década de los 90 del $1 \%$ en RTU y ninguno en incisión prostática (2). En realidad representa un inconveniente que retrasa 0 excepcionalmente excluye la posibilidad de realizar cualquier cirugía transuretral.

La detumescencia peneana, y por consiguiente el inicio de la cirugía endoscópica, se consigue con "trucos" mecánicos o medicamentosos.

\section{- Maniobras o trucos mecánicos}

- La más simple e inicial es suprimir cualquier manipulación sobre el pene ( pintura, tracción, intento de introducción del instrumento endoscópico, etc). Es conveniente acompañar esta "espera" con la colocación de bolsa con hielo sobre la raíz peneana.

- Si no conseguimos el efecto buscado, nosotros procedemos a la punción intracavernosa (bilateral) con agujas "mariposa" de reducido calibre; se consigue drenar lentamente la sangre retenida en cuerpos cavernosos.

Estas simples maniobras consiguen mayoritariamente la detumescencia peneana y permite iniciar la cirugía propuesta. En caso contrario proseguir con:

- Maniobras o trucos medicamentosos:

- Experimentalmente la fentolamina, como antagonista alfa-adrenoreceptor ha demostrado aumento del flujo arterial y relajación de la musculatura lisa con lo que se consigue la detumescencia; no hay experiencia suficiente. (3)

- Inyección intracavernosa de Eferina diluida ( $50 \mathrm{mls}$ en $10 \mathrm{mls}$ suero fisiológico).

- La inyección, previo clampeo, de $10 \mathrm{mg}$ de etilandrianol en la base de cuerpo cavernoso, con aguja 18/ 5 y masaje digital en el sitio de la inyección 
y desclampeo inmediato, consigue la detumescencia en el $88,4 \%$ de casos (4); debe excluirse esta maniobra en pacientes hipertensos y en todos los casos advertir al anestesista antes de proceder a esta maniobra.

\section{BIBUOGRAFíA RECOMENDADA}

1. VICENTE, J.R.: “Cirugía endoscópica de la hiperplasia benigna de próstata". Tratado de Endourología. J. Vicente R. Ed. Pulso, 303, 1996.

2. VICENTE, J.R.: "Tratamiento endoscópico: RTU/incisión prostática". HBP 2001. Ed. J. Vicente. Editorial Acción Médica, 153, 2001.

3. DIEDRICHS, W.; LUC, T.: "Reduction of sympathetic influences on penile erection by phentolamine". Urol. Int., 46: 64, 1991.

4. LÓPEZ, J.A.; PARISI, J.; SÁNCHEZ, G.: "Erección peneana postraquianestésica. Tratamiento con etiladrianol intracavernoso". Arch. Esp. Urol., 43: 241, 1990.

\section{TRUCOS EN PREVENCIÓN DE LA ESCLERO- SIS CERVICAL POST-RTU DE PRÓSTATA.}

J. Vicente Rodríguez.

La incidencia de la esclerosis cervical iatrógena varía según series: Mebust: $2.7 \%$, Roehborn: $1.7 \%$; en nuestra experiencia: $2.8 \%$ en la década de los 80 y $1.6 \%$ en la de los 90 .

TABLA I. RELACIÓ N VO LUMEN PRÓ STATA / ESC LERO SIS CERVIC AL

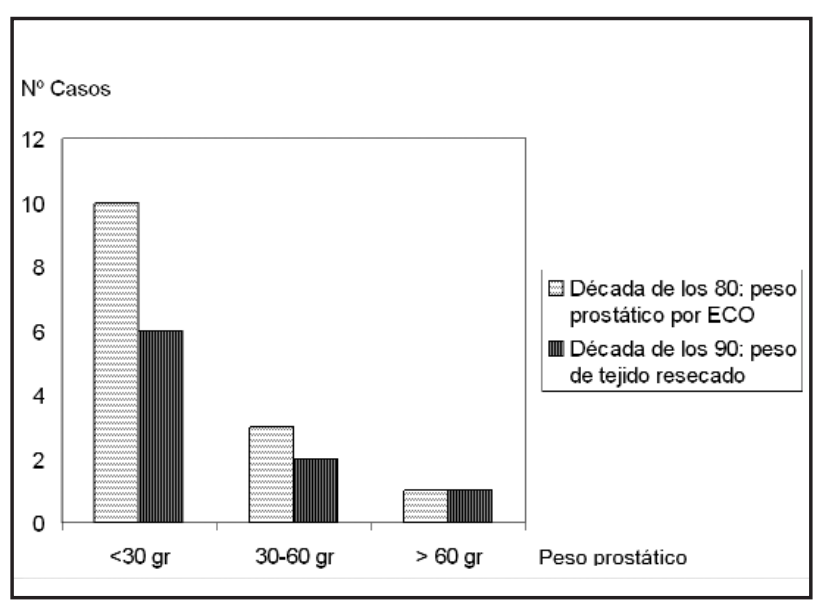

Desde los trabajos experimentales de Robinson y Green en 1962, se sabe que la RTU en próstatas pequeñas con escaso almohadillado cervical, son la principal causa de esclerosis cervical secundaria.

Trapasso en 1992 encontró que el 23\% de pacientes con tejido resecado inferior a 10 gramos, debieron ser reoperados por esclerosis cervical . En nuestra experiencia el $88 \%$ en la década de los 80 (1000 RTUs) y el 66 en la de los 90 (1482 c) de las esclerosis cervicales, correspondían a resecciones prostáticas menores de 30 gramos, como se refleja en la Tabla I.

Por otro lado, en próstatas pequeñas, si se realiza incisión prostática o prostatotomía, la incidencia de esclerosis cervical secundaria disminuye drásticamente: 0.4 \% para Roehborn o desaparece: $0 \%$ en nuestra experiencia.

Por consiguiente el TRUCO en la prevención de la esclerosis cervical secundaria (iatrógena) a la RTU, consiste en instituir la incisión prostática como maniobra quirúrgica desobstructiva en próstatas menores de 30 gramos y excluir en ellas la resección transuretral.

\section{TRUCOS EN PREVENCIÓN DE LA EYACU- LACIÓN RETRÓGRADA POST-RTU DE PRÓSTATA.}

J. Vicente Rodríguez.

La evidencia de la eyaculación retrógrada post RTU de la HBP varía según autores y series: desde el $93 \%$ para Malone, el 70.4 de Roehborn, $65.4 \%$ para Maderbacher hasta el $64.4 \%$ en nuestra experiencia.

LOS TRUCO S para prevenir o disminuir la incidencia de eyaculación retrógrada son:

- Próstatas pequeñas (menores de 30 gramos, bilobuladas, sin predominio del lóbulo medio) realizar incisión en vez de resección; así reducimos en este grupo de pacientes la incidencia de eyaculación retrógrada que fluctúa entre el $38.8 \%$ de Roehborn, el $22 \%$ en nuestra experiencia y el $4.1 \%$ de Maderbacher. 
- En próstatas de mediano / gran tamaño (entre 30-80 gramos) tributarias de resección transuretral la resección a nivel cervical debe alcanzar pero no sobrepasar las fibras del esfínter interno. Este se identifica endoscópicamente como fibras circulares, pálidas, paralelas entre sí y que aparecen a nivel cervical a distinta profundidad según el almohadillado adenomatino a nivel de cuello. Con esta precaución el esfínter interno queda respetado y la incidencia de eyaculación retrógrada disminuye; en nuestra serie clásica: $84.9 \%$, desciende a $64.4 \%$ en la actual.

\section{BIBUOGRAFÍA RECOMENDADA}

1. VICENTE, J.: “Tratado de Endourología”. Ed. Pulso, 5: 348, 1996.

2. VICENTE, J.: "HBP 2001”. Ed. Acción Médica, 4: 174, 2001.

\section{TRUCOS EN PREVENCIÓN DE LA INCONTI- NENCIA URINARIA YATRÓGENA POST-RTU DE PRÓSTATA.}

J. Vicente Rodríguez.

La incontinencia urinaria yatrógena post RTU, es infrecuente (incidencia 1\%) y asociada generalmente a la lesión esfinteriana peroperatoria, ligada casi siempre a defecto técnico en la ejecución de la resección prostática.

Esta incontinencia "vera" es necesario diferenciarla de la incontinencia transitoria de estrés y / 0 inestabilidad vesical: este es para Roehborn del 2.2\%, para Mebust del $1.7 \%$ y para nosotros $5.5 \%$, siendo la "verdadera" del $1 \%, 0.4$ y 0.94 respectivamente.

LOS TRUCOS para prevenir la incontinencia iatrogénica (postoperatoria y no ligada per se con vejiga inestable), están ligados a detalles técnicos.
- La colocación del paciente incorrecta altera el eje resección / axial del cuerpo alterando la identificación distal de resección; el paciente debe colocarse con las extremidades inferiores rotadas / anguladas 35 grados.

- El veru es el referente en el límite distal de la resección; sin embargo las masas apicales a veces le sobrepasan. Deberemos, para prevenir lesiones esfinterianas por resección distal, introducir el resector bajo visión y reconocer el esfínter externo por el signo de $\mathrm{N}$ esbit conociendo su relación con el veru, previo a la cirugía.

- La resección prolongada más distal al veru sucede al desplazar el instrumento (no el asa); para evitarlo deben realizarse (ayudando con la presión del dedo endorectal) los cortes en las masas apicales de corta longitud y de profundidad a superficie.

- Para evitar la resección profunda y distal de las masas apicales superiores: es recomendable colocar el resector con "visión de veru" y sin desplazar el instrumento rotarlo para realizar la resección en el hemicontorno superior.

- Una vez concluida la RTU de masas apicales inferiores y superiores, no se debe coagular los vasos apicales de forma indiscriminada y exagerada ya que se puede lesionar el tejido periesfinteriano.

- En próstatas menores de 30 gramos, la distancia cuello-veru es corta, con lo que el riesgo de resección prolongada distal, aumenta. En nuestra experiencia la mitad de las incontinencias acontecieron en pacientes con tejido resecado < 30 gr y ningún caso de incontinencia cuando en próstatas pequeñas realizamos incisión prostática. 